Para enlazar con este artículo / To link to this article:

http://dx.doi.org/10.6035/MonTI.2020.ne6.3

Para citar este artículo / To cite this article:

Koza, Walter \& Natalia Rivas Folch. (2020) "Modelización computacional de las locuciones verbales del español chileno a partir de la propuesta de la léxico-gramática." En: Mogorrón Huerta, Pedro (ed.) 2020. Análisis multidisciplinar del fenómeno de la variación fraseológica en traducción e interpretación / Multidisciplinary Analysis of the Phenomenon of Phraseological Variation in Translation and Interpreting. MonTI Special Issue 6, pp. 94-120.

\title{
MODELIZACIÓN COMPUTACIONAL DE LAS LOCUCIONES VERBALES DEL ESPAÑOL CHILENO A PARTIR DE LA PROPUESTA DE LA LÉXICO-GRAMÁTICA
}

\author{
COMPUTATIONAL MODELIZATION OF VERBAL IDIOMS OF \\ CHILEAN SPANISH BASED-ON LEXICON-GRAMMAR PROPOSAL
}

\author{
WALTER KOZA \\ walter.koza@pucv.cl \\ Pontificia Universidad Católica de Valparaíso \\ Proyecto FONDECyT 1171033 \\ NATALIA RIVAS FOLCH \\ natalia.rivas.f@gmail.com \\ Pontificia Universidad Católica de Valparaíso \\ Proyecto FONDECyT 1171033
}

\section{Resumen}

En el presente trabajo, se propone un estudio formal de las locuciones verbales (LV) pertenecientes al español de Chile con el propósito de diseñar un algoritmo computacional para el análisis automático. Para ello, se describen las LLVV incluidas en el Diccionario de uso del español de Chile, según los criterios de la léxico-gramática. Posteriormente, se procede a la modelización computacional recurriendo a NooJ, un programa que cuenta con diversas utilidades para el análisis lingüístico. Este método fue evaluado mediante el reconocimiento automático en un corpus compuesto por textos de prensa chilena. Se obtuvo 96,4\% de precisión, 92,23\% de cobertura y 94,53\% 
de medida F. Los resultados demuestran que el algoritmo diseñado es adecuado para el análisis automático de las LLVV.

Palabras clave: Locuciones verbales; Español chileno; Análisis automático; léxicogramática; NooJ.

\begin{abstract}
In this work, a formal study for Verbal Idioms (VI) of Chilean Spanish is proposed with the objective of developing a computational algorithm for the automatic analysis of texts. For this, the VIS included in Diccionario de uso del español de Chile were described according to the lexicon-grammar theory. Then, a computational modelization was made using NooJ, a program that has several utilities. This method was evaluated with an automatic recognition in a corpus composed by texts from Chilean press. $96.4 \%$ of precision, $92.23 \%$ of coverage and $94.53 \%$ of F-measure were obtained. The results show that the algorithm designed is adecuated for the automatic analysis of the VI.
\end{abstract}

Keywords: Verbal idioms; Chilean Spanish; Automatic Analysis; Lexicon-grammar; NooJ.

\title{
1. Introducción
}

Uno de los problemas más relevantes que ha venido enfrentando la lingüística computacional ha sido establecer un modo efectivo para el análisis de las locuciones (Vietri 2014, Silberztein 2016). Las complejidades que implican estas expresiones para el análisis automático tienen que ver, fundamentalmente, con el grado de fijación, pues, mientras algunas locuciones no permiten separar sus elementos, otras sí lo hacen, como en (1). A su vez, otro inconveniente se deriva de casos en los que es posible modificar uno de sus miembros (2). Y ya en el caso de las locuciones verbales, un desafío lo constituye el hecho de que el verbo puede adquirir condiciones sintácticas que no posee cuando aparece de manera libre (3):

(1) Juan agarra para el hueveo a María. --> Juan agarra a María para el hueveo.

(2) Juan dejó la escoba --> Juan dejó la mansa escoba.

(3) Juan tiene una casa / *La casa es tenida por Juan. --> Juan tiene en cuenta a María. / María es tenida en cuenta por Juan. 
A tales efectos, en el presente trabajo se propone un análisis de las locuciones verbales (LLVV) pertenecientes al español de Chile con el propósito de diseñar un algoritmo computacional para el análisis automático de textos del lenguaje natural. Con este objetivo, se busca realizar una descripción exhaustiva del comportamiento morfosintáctico que poseen estos elementos tomando como fundamentos teóricos y metodológicos, los planteamientos de la léxico-gramática (Gross 1975; 1984). Para ello, se analizan las LLVV listadas en el Diccionario de uso del español de Chile (DUECh) (Academia Chilena de la Lengua 2010), categorizándolas en diversas clases, según su estructura y en relación con la exigencia de argumentos y posibilidades transformacionales.

La fase anteriormente descrita es modelizada computacionalmente. Esta labor se realiza con el software de acceso libre NooJ, desarrollado por Silberztein $(2003 ; 2016)$. Este programa presenta una estructura basada en la jerarquía de Chomsky-Schützenberger (Chomsky 1957) y cuenta con diversas utilidades para el análisis lingüístico. El trabajo computacional implicó la construcción de un diccionario electrónico de LLVV y gramáticas sintácticas. Este método fue evaluado mediante el reconocimiento automático en un corpus compuesto por textos de prensa chilena, logrando 96,4\% de precisión, $92,23 \%$ de cobertura y $94,53 \%$ de medida F. Estos resultados demuestran que el algoritmo diseñado es adecuado para el análisis automático de las LLVV.

\section{Marco teórico}

En este apartado se presenta el marco teórico que sustenta la investigación. Se incluyen, por un lado, los trabajos referentes a la naturaleza de la locución y, por otro lado, la propuesta de la léxico-gramática (Gross 1975; 1984). Dentro de esta última se hace mención al trabajo de Vietri (2014), quien lleva a cabo un análisis de las LLVV del italiano, el cual, al igual que en este estudio, implica la creación de un algoritmo computacional en NooJ.

\subsection{Respecto al concepto de locución}

En el sistema de la lengua es posible encontrar, además de combinaciones libres de palabras, ciertas estructuras fijas que poseen mayor estabilidad. Se trata de estructuras que, según Corpas Pastor (1996: 18): 
i. Constan de al menos dos palabras ortográficas.

ii. Presentan cierto grado de lexicalización.

iii. Se caracterizan por la alta frecuencia de coaparición en la lengua.

Estas construcciones son denominadas por la autora como unidades fraseológicas (UF) y cuentan con una tradición de estudios en la lingüística. Dentro de este tipo de expresiones, se incluyen las locuciones, entendidas, según la propuesta de Casares (1950) reformulada por Penadés (2012), como "la combinación fija de palabras que funciona como elemento de la oración y cuyo significado no se corresponde con la suma de los significados de sus componentes" (Penadés 2012:25).

El criterio de clasificación de las locuciones más utilizado remite a la categoría gramatical que se identifica según su función sintáctica. De este modo, se establecen locuciones nominales, adjetivales, preposicionales, adverbiales y verbales (Zuluaga 1975; Corpas Pastor 1996; Penadés 2012). Las LLVV contienen un verbo, el cual forma un predicado complejo a partir de la asimilación de un complemento o adjunto. Esto implica que: (i) van a exigir argumentos; (ii) están supeditadas a las restricciones de concordancia mediante la flexión; (iii) pueden designar estados y eventos (Silvagni 2017); (iv) pueden ser modificadas por un adjunto.

Ahora bien, en relación con el ítem (i), para la identificación de argumentos exigidos por las LLVV, es necesario tener en cuenta las restricciones que la locución le impone a sus argumentos. Así, por ejemplo, 'calentar' en 'calentar el asiento' solo exige un único argumento que debe tener el rasgo +humano, dado que presenta a un complemento directo cristalizado:

(4) Juan calentó el asiento.

*El sol calentó el asiento.

Asimismo, también es pertinente observar las posibilidades transformacionales de las LLVV, como la voz pasiva, la nominalización, etcétera.

(5) Juan agarra a María para el hueveo = María fue agarrada para el hueveo por Juan.

Pedro agachó el moño = Pedro tuvo una agachada de moño. 
Para una descripción exhaustiva tanto de la naturaleza de los argumentos como de las posibilidades transformacionales de las LLVV, se recurrió al marco de la léxico-gramática, cuyos principales postulados se presentan en la sección siguiente.

\subsection{La léxico-gramática}

La léxico-gramática (Gross 1975; 1984) se presenta como un método de descripción de las lenguas naturales basado en tres aspectos principales: (i) la sintaxis es indisoluble del lexicón; (ii) la unidad mínima de análisis es la oración simple (a diferencia de palabras o sintagmas), y (iii) el marco debe ofrecer una formalización y un método descriptivo aplicable a cualquier lengua (Elia, Monteleone \& Marano 2011). Este modelo plantea un estudio combinado de las reglas sintácticas y preferencias de selección léxica en relación con las posibilidades de transformación que tiene una oración determinada.

Para poder llegar a tal descripción, Gross (1975) propone la elaboración de tablas organizadas en clases, las cuales reúnen elementos léxicos de una categoría gramatical que comparten propiedades (Tolone 2012). Una tabla se presenta sobre la forma de una matriz, la cual contiene: (i) en línea, los elementos de la clase correspondiente; (ii) en columna, las propiedades sintáctico-semánticas (que no son necesariamente aceptadas por todos los miembros de la clase), y (iii) al cruce de una línea y de una columna, el signo + o -, en función de si la entrada léxica descrita por la línea acepta o no la propiedad descrita por la columna. Una propiedad sintáctico-semántica es una información que se refiere directamente a la construcción de base asociada a la clase, a una transformación de la construcción de base o a una construcción adicional. Si una unidad léxica tiene dos sentidos distintos, entonces presentará dos entradas. A modo de ejemplo, en la tabla 1 se muestra un fragmento de una de las tablas de verbos del francés adaptadas por Pivaut (1989), presentada en Tolone (2012). 


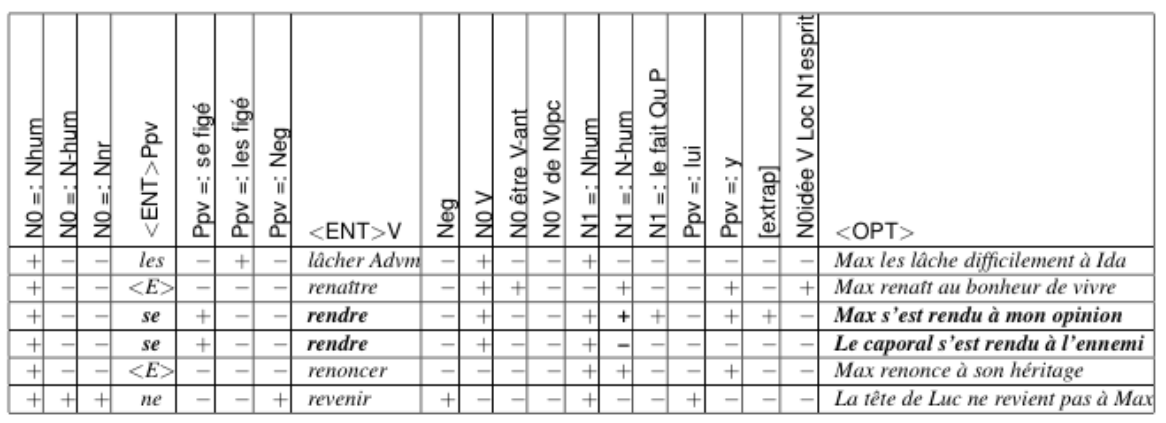

Tabla 1. Ejemplo de tabla Léxico-Gramática (Tolone 2012)

Como se puede observar, un verbo como rendre ('rendir') va a tener doble entrada según los tipos de objetos que exija (NO=Humano, N1=No Humano: 'Max se rindió a mi opinión'; N0=Humano, N1=Humano: 'El cabo se rindió al enemigo').

Posteriormente, se identifican las transformaciones que admite cada oración ilustrada en la última columna $(<\mathrm{OPT}>)$. La noción de transformación es propuesta por Harris (1970) y alude a las diversas posibilidades en las que puede proyectarse una estructura argumental. Esta idea no debe confundirse con la que se plantea en la gramática generativa transformacional, puesto que desde la perspectiva harrisiana, todas las transformaciones tendrían lugar en la estructura superficial. En el ejemplo presentado se puede apreciar que las dos oraciones admiten la nominalización ('Tuvo lugar la rendición de Max ante mi opinión', 'Tuvo lugar la rendición del cabo ante el enemigo').

En relación con las expresiones idiomáticas, Gross (1975) señala que esta metodología puede extenderse a este tipo de expresiones. En relación con ello, Vietri (2014) realiza un análisis de las LLVV del italiano a partir de este aparato teórico-metodológico. Para llevar a cabo sus descripciones, Vietri (2014) parte de la introspección y juicios lingüísticos, corroborando luego estos dos criterios con información proveniente de Internet. Una vez realizada la descripción de las unidades, la autora da cuenta de las posibilidades que introduce la léxico-gramática para poder realizar una formalización de las expresiones idiomáticas descritas. A tales efectos, emplea el software NooJ y crea gramáticas que le permiten detectar las propiedades de las unidades, con las respectivas variaciones que estas admiten. 
En este sentido, algunas de las conclusiones a las que llega Vietri (2014) son: (i) las LLVV se rigen por las mismas reglas sintácticas que los verbos predicativos; (ii) respecto de las posibilidades de descomposición de una locución, esto debe verse caso por caso; (iii) la representación semántica de las expresiones idiomáticas se encuentra directamente ligada con su sintaxis; (iv) el uso de la léxico-gramática constituye un marco adecuado para realizar una descripción exhaustiva de unidades como las estudiadas considerando la posterior formalización de reglas para la modelización computacional.

Aquí se toma como base la propuesta de esta autora para las LLVV del español chileno. Al igual que aquella, se realiza una descripción morfosintáctica de estas unidades, incluyendo sus posibilidades combinatorias a través de la estructura argumental que proyectan. Asimismo, se considerarán sus posibilidades transformacionales (nominalización, voz pasiva, etcétera). Posteriormente, se propondrá una formalización y una modelización computacional para la detección automática en textos de lenguaje natural.

\section{Metodología}

En este apartado se presentan los lineamientos metodológicos que sustentan esta investigación. Se compone de dos secciones, la primera de ellas describe la naturaleza de las LLVV y la segunda, el trabajo computacional.

\subsection{Descripción de las LLVV}

La descripción se realizó sobre una lista de LLVV extraída del DUECh (Academia Chilena de la Lengua 2010). Este diccionario concentra una serie de entradas léxicas o lemas que remiten a expresiones usadas en el habla de Chile. De acuerdo con Matus, director del diccionario, la obra se propone reflejar el uso corriente, socialmente estabilizado del habla de dicho país (Sáez 2012). En este sentido, primó un criterio lexicográfico en el que se concibió como LV a toda expresión indicada de esa manera en el DUECh, más allá de ciertas omisiones señaladas por Sáez (2012), como el hecho de ser omisiones. Ahora bien, el presente trabajo pretende dar cuenta de un método para describir morfosintácticamente cualquier expresión fija conformada por un verbo más argumentos o adjuntos cristalizados, por lo cual se podría aplicar a nuevas unidades léxicas de este tipo que se detecten o se creen en el futuro. 
El primer paso fue la extracción de todas aquellas entradas que pertenecen a la categoría de "locución verbal", y se recopiló un total de 833 expresiones de este tipo. Posteriormente se las clasificó en dos grandes grupos; el primero incluye a las LLVV que no expresaban negación ('agachar el moño') y el segundo, las que sí lo hacen ('no ver una').

\begin{tabular}{|l|c|c|}
\hline & Total lemas & Porcentaje \\
\hline Locuciones Grupo 1 & 792 & $95,1 \%$ \\
\hline Locuciones Grupo 2 & 41 & $4,9 \%$ \\
\hline Locuciones totales (G1 + G2) & 833 & $100 \%$ \\
\hline
\end{tabular}

Tabla 2. Resumen de locuciones extraídas del DUECh

En la tabla 2 se puede apreciar un resumen del total de locuciones extraídas del DUECh. En el presente trabajo se realizó una descripción del primer grupo, quedando para futuras investigaciones el segundo.

Posterior a la recopilación se llevó a cabo una categorización y descripción de estas estructuras según los criterios que plantea la léxico-gramática (Gross $1975 ;$ 1984). A continuación, se detalla el procedimiento.

\subsubsection{Análisis distribucional}

En una primera instancia se tomó en consideración el modo en el que se estructura el lema en el diccionario, esto es, se observaron los elementos constitutivos de la locución. De este modo, expresiones como 'dejar la escoba' y 'sacarse el pillo' fueron agrupadas en clases diferentes, dado que una requiere de clítico y la otra no. Sin embargo, al realizar las tablas se pudo observar que ciertas locuciones poseen requerimientos argumentales diferentes al contemplado inicialmente. Así, aquellas locuciones declaradas en el DUECh con pronombre enclítico, como por ejemplo, "meterse en un queso" podían emplearse sin él en casos como (4):

(4) Juan metió en un queso a María.

De la misma manera, en locuciones cuyo lema aparece sin el pronombre, como en "sacar en cara", su uso sí lo exige:

(5) María te sacó en cara todo lo que había hecho por ti. 
Este análisis arrojó las siguientes clases:

\begin{tabular}{|c|c|c|c|c|}
\hline Clase & $\begin{array}{l}\text { Ejemplo de } \\
\text { lema }\end{array}$ & Ejemplo en uso & Total & $\%$ \\
\hline $\mathrm{Cl} 1:(\mathrm{NO})+\mathrm{V}+\mathrm{Cl}$ & echar al agua. & $\begin{array}{l}\text { María echó al } \\
\text { agua a Juan. }\end{array}$ & 405 & 51,14 \\
\hline $\mathrm{Cl} 2:(\mathrm{NO})+\mathrm{CL}+\mathrm{V}+\mathrm{Cl}$ & $\begin{array}{l}\text { arrancarse con } \\
\text { los tarros. }\end{array}$ & $\begin{array}{l}\text { Juan se arrancó } \\
\text { con los tarros. }\end{array}$ & 287 & 36,24 \\
\hline $\mathrm{Cl} 3:(\mathrm{NO})+\mathrm{CL}+\mathrm{CL}+\mathrm{V}+\mathrm{Cl}$ & $\begin{array}{l}\text { sabérselas por } \\
\text { libro. }\end{array}$ & $\begin{array}{l}\text { Ella jura que } \\
\text { se las sabe por } \\
\text { libro. }\end{array}$ & 6 & 0,76 \\
\hline $\mathrm{Cl} 4:(a)+(\mathrm{N} 0)+C L d a t+V$ & $\begin{array}{l}\text { crujirle a } \\
\text { alguien. }\end{array}$ & $\begin{array}{l}\text { A Juan le crujió } \\
\text { por fin. }\end{array}$ & 6 & 0,76 \\
\hline $\mathrm{Cl} 5:(\mathrm{a})+(\mathrm{N} 0)+\mathrm{CLdat}+\mathrm{V}+\mathrm{Cl}$ & $\begin{array}{l}\text { faltarle a } \\
\text { alguien } \\
\text { chauchas para } \\
\text { el peso. }\end{array}$ & $\begin{array}{l}\text { A María le faltan } \\
\text { chauchas para el } \\
\text { peso. }\end{array}$ & 35 & 4,42 \\
\hline $\mathrm{Cl}$ 6: (a) $+(\mathrm{NO})+\mathrm{se}+\mathrm{CLdat}+\mathrm{V}+\mathrm{Cl}$ & $\begin{array}{l}\text { aconchársele } \\
\text { a alguien los } \\
\text { meados. }\end{array}$ & $\begin{array}{l}\text { A Juan se le } \\
\text { aconcharon los } \\
\text { meados, por eso } \\
\text { no reacciona. }\end{array}$ & 40 & 5,05 \\
\hline $\mathrm{Cl} 7:(a)+(\mathrm{NO})+\mathrm{se}+\mathrm{CLdat}+\mathrm{V}$ & $\begin{array}{l}\text { chupársele a } \\
\text { alguien. }\end{array}$ & $\begin{array}{l}\text { A María se } \\
\text { le chupa ir a } \\
\text { hablarle. }\end{array}$ & 3 & 0,38 \\
\hline $\begin{array}{l}\mathrm{Cl} 8: \\
\text { (a)+(N0masc }+ \text { CLdat }+\mathrm{V}+\mathrm{Cl}\end{array}$ & $\begin{array}{l}\text { gustarle a } \\
\text { un hombre } \\
\text { las patitas de } \\
\text { chancho. }\end{array}$ & $\begin{array}{l}\text { Está claro que a } \\
\text { Juan le gustan } \\
\text { las patitas de } \\
\text { chancho. }\end{array}$ & 2 & 0,25 \\
\hline $\begin{array}{l}\mathrm{Cl} 9: \\
(\mathrm{a})+(\mathrm{NOmasc})+\mathrm{se}+\mathrm{CLdat}+\mathrm{V}+\mathrm{Cl}\end{array}$ & $\begin{array}{l}\text { quemársele a } \\
\text { un hombre el } \\
\text { arroz. }\end{array}$ & $\begin{array}{l}\text { María no sabía } \\
\text { que a Juan se } \\
\text { le quemaba el } \\
\text { arroz. }\end{array}$ & 7 & 0,88 \\
\hline $\mathrm{Cl}$ 10: (a)+(NOfem $)+C L+V+C l$ & $\begin{array}{l}\text { dejar a una } \\
\text { mujer el tren. }\end{array}$ & $\begin{array}{l}\text { A María la dejó } \\
\text { el tren de tanto } \\
\text { esperar a Juan. }\end{array}$ & 1 & 0,13 \\
\hline
\end{tabular}

Tabla 3. Resumen de categorización de locuciones pertenecientes al primer grupo.

En la tabla anterior, es posible observar las diversas categorías que emergieron a partir de los diversos requerimientos argumentales que poseen las 
locuciones. En cada una de las clases se da cuenta de la organización prototípica de la expresión al emplearse. Asimismo, cada categoría contempla una serie de elementos que contiene la locución:

\begin{tabular}{|c|l|}
\hline Elemento & \multicolumn{1}{c|}{ Significado } \\
\hline (a) & Introduce el complemento indirecto en la locución. \\
\hline (N0) & $\begin{array}{l}\text { Primer argumento exigido por la locución, que puede tener tanto función } \\
\text { de sujeto como de complemento indirecto. Un No puede ser tácito y no } \\
\text { tener realización fonológica, esta particularidad se indica entre paréntesis. }\end{array}$ \\
\hline le/se & $\begin{array}{l}\text { Dan cuenta de los pronombres clíticos determinados o definidos por una } \\
\text { locución en particular. En estos casos, la expresión únicamente admite } \\
\text { estas opciones de clíticos. }\end{array}$ \\
\hline CL & $\begin{array}{l}\text { Indica que en esa parte de la locución se puede insertar un pronombre } \\
\text { clítico dativo o acusativo. Sin embargo, algunas locuciones exigen un } \\
\text { tipo particular de pronombre; en esos casos, se indica en la clase como } \\
\text { "CLdat", pues en aquel lugar solo se admiten pronombres dativos. }\end{array}$ \\
\hline V & $\begin{array}{l}\text { Forma verbal que debe ser conjugada. Se constituye como el núcleo de } \\
\text { la locución. }\end{array}$ \\
\hline C1 & Representa la parte "invariable" de la locución. \\
\hline
\end{tabular}

Tabla 4. Descripción de los elementos constitutivos por cada clase.

Posteriormente al trabajo de categorización se prosiguió con la confección de tablas léxico-gramática para cada clase. Estas permiten contar con una base de datos de los distintos argumentos de las LLVV. Respecto de la estructura de las tablas, se siguen los lineamientos de Gross (1984) y Vietri (2014). A continuación, se ejemplifica con un fragmento del análisis distribucional de las LLVV de la clase 5.

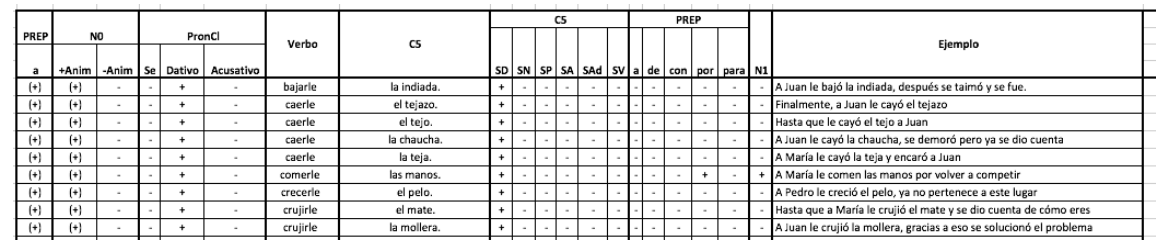

Tabla 5. Ejemplo tabla léxico-gramática de la clase 5.

Como se puede apreciar, las tablas léxico-gramática poseen forma de matriz, en cuyas líneas se define cada una de las locuciones y en sus columnas se 
precisan las diversas propiedades sintáctico-semánticas. A continuación, se sintetiza la cantidad de LLVV que incluye cada clase.

\begin{tabular}{|c|c|c|}
\hline Clase & Total locuciones & $\begin{array}{c}\text { Total } \\
\text { Entradas }\end{array}$ \\
\hline $\mathrm{Cl} \mathrm{l}:(\mathrm{NO})+\mathrm{V}+\mathrm{Cl}$ & 405 & 798 \\
\hline $\mathrm{Cl} 2:(\mathrm{NO})+\mathrm{CL}+\mathrm{V}+\mathrm{C} 2$ & 287 & 459 \\
\hline $\mathrm{Cl} 3:(\mathrm{NO})+\mathrm{CL}+\mathrm{CL}+\mathrm{V}+\mathrm{C} 3$ & 6 & 12 \\
\hline $\mathrm{Cl} 4:(\mathrm{a})+(\mathrm{NO})+\mathrm{CLdat}+\mathrm{V}$ & 6 & 6 \\
\hline $\mathrm{Cl} 5:(\mathrm{a})+(\mathrm{NO})+\mathrm{CLdat}+\mathrm{V}+\mathrm{C} 5$ & 35 & 36 \\
\hline $\mathrm{Cl}$ 6: (a)+(NO)+se+CLdat+V+C6 & 40 & 40 \\
\hline $\mathrm{Cl} 7:(\mathrm{a})+(\mathrm{NO})+\mathrm{se}+\mathrm{CLdat}+\mathrm{V}$ & 3 & 4 \\
\hline $\mathrm{Cl}$ 8: (a)+(N0masc) $+\mathrm{CLdat}+\mathrm{V}+\mathrm{C} 8$ & 2 & 2 \\
\hline $\mathrm{Cl}$ 9: (a)+(NOmasc)+se+CLdat+V+C9 & 7 & 7 \\
\hline $\mathrm{Cl}$ 10: (a)+(NOfem)+CL+V+Cl0 & 1 & 2 \\
\hline
\end{tabular}

Tabla 6. Comparación cantidad de lemas por clase versus cantidad de entradas en tabla léxico-gramática

Puede observarse que las primeras clases poseen una gran diferencia entre la cantidad de lemas y las entradas en el diccionario. Esto porque, si se piensan las clases en un continuum, en el que la 1 es la más simple y la 10 la más compleja, se puede vislumbrar que, a medida que se van complejizando las clases, los usos o las propiedades que admiten para su empleo se restringen, lo cual se traduce en una reducción de la cantidad de entradas en las tablas.

\subsubsection{Transformaciones}

Posteriormente, se llevó a cabo la tabla de transformaciones. En el presente estudio se identificaron trece; sin embargo, se tiene como proyección expandir esta lista. Se resume cada transformación en la siguiente tabla: 


\begin{tabular}{|c|c|c|}
\hline Transformación & Significado & Ejemplo \\
\hline 1 & Negación con "no" & $\begin{array}{l}\text {-dar bola } \\
\text { Ella le dio bola = Ella no le dio bola. }\end{array}$ \\
\hline 2 & Negación con "sin" & $\begin{array}{l}\text {-hacer bolsa } \\
\text { Ella hizo bolsa sus juguetes = Sin que } \\
\text { haga bolsa tus juguetes. }\end{array}$ \\
\hline 3 & Voz pasiva & $\begin{array}{l}\text {-hacer bolsa } \\
\text { María hizo mierda el auto = El auto } \\
\text { fue hecho mierda por María. }\end{array}$ \\
\hline 4 & $\begin{array}{l}\text { Reemplazo de } \mathrm{Cl} \text { por } \\
\text { clítico }\end{array}$ & $\begin{array}{l}\text {-pegar la PLR } \\
\text { María le pegó la PLR= María se la } \\
\text { pegó. }\end{array}$ \\
\hline 5 & $\begin{array}{l}\text { Reemplazo de } \mathrm{Al} \text { por un } \\
\text { clítico acusativo }\end{array}$ & $\begin{array}{l}\text {-hacer mierda } \\
\text { Juan hizo mierda a María = Juan la } \\
\text { hizo mierda }\end{array}$ \\
\hline 6 & Pluralización de los clíticos & $\begin{array}{l}\text {-dejarle la cagada } \\
\text { María le dejó = María les dejó la } \\
\text { cagada. }\end{array}$ \\
\hline 7 & Interrogación & $\begin{array}{l}\text {-Dejar la escoba } \\
\text { Juan dejó la escoba = ¿Quién dejó la } \\
\text { escoba? }\end{array}$ \\
\hline 8 & Condicional & $\begin{array}{l}\text {-Quedar pegado } \\
\text { Juan quedó pegado = si Juan quedó } \\
\text { pegado, María también. }\end{array}$ \\
\hline 9 & Cl con flexión de número & $\begin{array}{l}\text {-Estar cagado } \\
\text { Él está cagado = Ellos están cagados. }\end{array}$ \\
\hline 10 & Cl con flexión de género & $\begin{array}{l}\text {-hacer huevón } \\
\text { Él lo hizo huevón= Él la hizo } \\
\text { huevona. }\end{array}$ \\
\hline 11 & $\mathrm{Cl}$ con diminutivo & $\begin{array}{l}\text {-hacer tuto } \\
\text { Ellos hicieron tuto = Ellos hicieron } \\
\text { tutito. }\end{array}$ \\
\hline 12 & C con aumentativos & $\begin{array}{l}\text {-caer gordo } \\
\text { Él le cayó gordo= Él le cayó } \\
\text { gordísimo. }\end{array}$ \\
\hline 13 & $\begin{array}{l}\text { Inclusión de un } \\
\text { modificador de } \mathrm{Cl}\end{array}$ & $\begin{array}{l}\text {-Quedar la cagada } \\
\text { Ayer quedó la cagada = Ayer quedó la } \\
\text { mansa cagada. }\end{array}$ \\
\hline
\end{tabular}

Tabla 7. Transformaciones de locuciones verbales del español de Chile 
En este punto es pertinente aclarar que las transformaciones 11, 12 y 13 podrían corresponderse con lo que se considera desautomatización (Zuluaga 2001; Mena Martínez 2003). Este concepto alude al fenómeno de desviación que hace un hablante de una unidad fraseológica de su forma canónica, como ejemplifica Mena Martínez (2003): 'dar gato por euro' en lugar de 'dar gato por liebre'. Generalmente, esto se realiza por motivos expresivos, humorísticos, etcétera, lo que se correspondería con lo que se considera la actuación, lo cual excede los objetivos del presente trabajo. No obstante, se va a tener en cuenta los tipos de desautomatización descritos en 11,12 y 13 como transformaciones, puesto que se mantiene el contenido semántico de la LV.

Una vez elaborado el glosario de las transformaciones, se prosiguió con la confección de las tablas. Se presenta un fragmento de las transformaciones de la clase 1 .

\begin{tabular}{|c|c|c|c|c|c|c|c|c|c|c|c|c|c|c|}
\hline \multirow{2}{*}{ Verbo } & \multirow{2}{*}{$\mathrm{C} 1$} & \multirow{2}{*}{ Ejemplo } & \multicolumn{12}{|c|}{ Transformaciones } \\
\hline & & & $\mathrm{TI}$ & $\mathrm{T} 2$ & $\mathrm{T3}$ & T4 & TS & 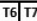 & $7 \mathrm{~T}$ & $\mathrm{~T} 9$ & $\mathrm{~T} 10 \mathrm{~T}$ & T11 & $\mathrm{T} 12$ & $T 13$ \\
\hline hacer & mierda. & Ellos hicieron mierda todo lo que provenía de sus enemigos & + & + & + & -1 & + & $-4+$ & + & -1 & -1 & - & -1 & - \\
\hline hacer & mierda. & La podadora hizo mierda todas las flores & + & + & + & - & + & - & + & - & - & - & - & - \\
\hline hacer & mierda. & Ellos se harán mierda la ropa si siguen peleando & + & + & - & - & + & - & + & - & - & - & - & - \\
\hline hacer & mierda. & Viene el aluvión y los carteles se harán mierda con la tormenta & + & + & 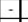 & - & - & - & + & - & - & - & - & - \\
\hline hacer & mierda. & Pamela me hará mierda las uñas & + & + & -1 & - & + & $-1+$ & + & - & - & - & - & - \\
\hline hacer & mierda. & Las espinas me harán mierda la ropa & + & + & 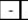 & -1 & +1 & $-1+$ & + & $|-|$ & - & - & - & - \\
\hline
\end{tabular}

Tabla 8. Ejemplo transformaciones locución Clase 1

En la tabla anterior se detalla, en primera instancia, los componentes nucleares de la locución $(\mathrm{V}+\mathrm{C})$ y, posteriormente, las transformaciones que admite, lo cual, al igual que en las tablas léxico-gramática, se va indicando con un signo + si la expresión admite determinada modificación o con un - si no es así. Tomando como ejemplo el caso de la tabla 8 , se puede señalar que la oración 'Ellos hicieron mierda todo lo que provenía de sus enemigos' admite:

\begin{tabular}{|c|l|}
\hline Transformación & <OPT $>$ \\
\hline T1 & Ellos no hicieron mierda todo lo que provenía de sus enemigos \\
\hline T2 & Sin que ellos hicieran mierda todo lo que provenía de sus enemigos. \\
\hline T3 & Todo lo que provenía de sus enemigos fue hecho mierda por ellos. \\
\hline T5 & Ellos lo hicieron mierda \\
\hline T7 & ¿Quiénes hicieron mierda todo lo que provenía de sus enemigos? \\
\hline T8 & Si ellos hicieron mierda todo lo que provenía de sus enemigos... \\
\hline
\end{tabular}

Tabla 9. Ejemplo transformaciones 
Como se puede observar, la locución descrita no admite todas las transformaciones, por lo que, por ejemplo, construcciones como (6) resultarán agramaticales.

(6) a. *Ellos los hicieron (T4)

b. *Ellos hicieron mierdas (T9)

Una vez realizados estos procedimientos, que permiten proponer una exhaustiva descripción de las LLVV del español de Chile, se procedió a una modelización computacional en NooJ.

\subsection{Trabajo computacional}

Para llevar a cabo el procedimiento de detección automáticas de las LLVV del español de Chile fue necesario formalizar las estructuras que posee cada clase. Se recurrió a NooJ, una herramienta que cuenta con diversas utilidades para el tratamiento de lenguaje natural y que se puede organizar a partir de la jerarquía propuesta por Chomsky y Schützenberger (1963). El programa cuenta con los siguientes recursos:

- diccionarios: listas de palabras con diversos tipos de información lingüística.

- gramáticas morfológicas y derivacionales: modelos de flexión y derivación.

- gramáticas productivas: sistemas regulares o gráficos útiles para el tratamiento de cadenas de caracteres con determinadas propiedades formales.

- gramáticas sintácticas (archivos .nog): sistemas regulares o gráficos útiles para el tratamiento de cadenas de caracteres formadas por dos o más unidades léxicas, generalmente, separadas por espacios en blanco.

Como se ha mencionado, la lista de palabras del diccionario contempla información de diversa índole, según sean los intereses del usuario (morfológica, sintáctica, léxica, semántica, etcétera). A su vez, los lemas pueden asociarse a modelos morfológicos que permiten la flexión automática. Por ejemplo, una palabra como 'médico' está listada de la siguiente manera. 
médico,N+anim+hum+pm+FLX=NIÑ

Eso significa que 'médico' es un nombre ('N') que posee los rasgos 'animado' ('+anim'), 'humano' ('+hum') y 'profesional de la medicina' ('pm'), y tiene una flexión ('FLX') correspondiente con el modelo NIÑO, especificado en la gramática morfológica:

$\mathrm{NINO}=<\mathrm{E}>/$ masc + sg $\mid<\mathrm{B}>\mathrm{s} /$ masc + pl $|<\mathrm{B}>\mathrm{a} / \mathrm{fem}+\mathrm{sg}|<\mathrm{B}>$ as/fem + pl $;$

Con dicha gramática es posible generar automáticamente 'médico', 'médicos', 'médica', 'médicas'. Este procedimiento resulta mucho más eficaz y económico, debido a que el mismo modelo permite flexionar numerosas palabras ('médico', 'enfermero, 'farmacéutico', etcétera); en el caso de los verbos en español, que tienen una enorme riqueza flexiva, las ventajas de este tipo de gramáticas son indiscutibles (Bonino 2015).

A su vez, también el programa cuenta con la posibilidad de elaborar gramáticas de diversos niveles (dependientes del contexto, de estados finitos, etcétera). Por ejemplo, como se puede apreciar en Silberztein (2016), una gramática sencilla para el SN del inglés puede esquematizarse de la siguiente manera:

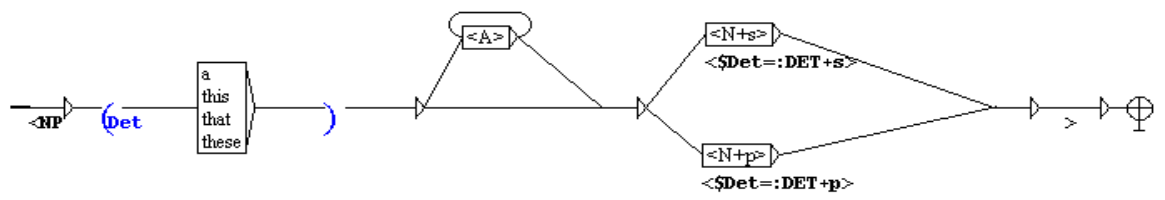

Figura 1. Ejemplo de una gramática sintáctica NooJ para SN del inglés

Como indica la figura 1, la gramática comienza con un estado inicial compuesto por un determinante, indicado entre paréntesis, puede incluir un adjetivo $(<\mathrm{A}>)$ y finalizar en el núcleo nominal $(<\mathrm{N}>)$. A la vez, se especifica la regla de concordancia de número: si $\mathrm{N}$ tiene el rasgo singular (' $+\mathrm{s}$ '), entonces el número del determinante será singular; en cambio, si el $\mathrm{N}$ posee el rasgo plural, el determinante tomará ese mismo rasgo. De este modo, cada expresión que contenga la información declarada será etiquetada como NP (Noun Phrase) $(<\mathrm{NP} \ldots>)$. Estas gramáticas pueden operar tanto en el nivel 
sintáctico como en el morfológico y permiten reconocer y generar de manera automática.

En esta ocasión se siguieron los siguientes procedimientos: (i) elaboración de gramáticas léxicas; (ii) confección de un diccionario que contemplara todas las propiedades de cada clase de locución.

\subsubsection{Elaboración de gramáticas y diccionarios}

Cada una de las gramáticas elaboradas presenta como núcleos básicos: (i) el nombre de la clase de locución; (ii) la unidad V, que indica el núcleo verbal de la expresión, y (iii) los elementos que componen la parte "fija" de la expresión. De esta manera, la gramática presenta estructuras como las que se ejemplifican en la tabla 10.

\begin{tabular}{|l|c|c|c|c|c|}
\hline \multicolumn{1}{|c|}{ Ejemplo } & V & PREPC & CONT & DETC & C \\
\hline hacer mierda & Hacer & - & - & - & mierda \\
\hline dejar la escoba & Dejar & - & - & la & escoba \\
\hline atornillar al revés & Atornillar & - & al & - & revés \\
\hline caer de cajón & Caer & de & - & - & cajón \\
\hline agarrar para el chuleteo & Agarrar & para & - & el & chuleteo \\
\hline
\end{tabular}

Tabla 10. Ejemplo composición de elementos centrales de una locución verbal del español

El número en el elemento $C$ cambiará según cada clase. Por ejemplo, para la clase 1, se denomina $\mathrm{C} 1$ y para la clase 2, C2 y así sucesivamente.

Las gramáticas irán variando según sus elementos constitutivos y, para evidenciarlo, se ejemplificará con las gramáticas de clase 1 y clase 2 . En la figura 2 se muestra la primera de ellas.

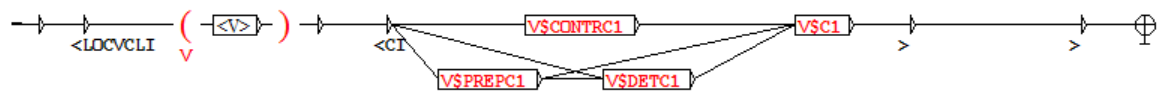

Figura 2. Gramática léxica para detectar locuciones clase 1 
En esta figura se pueden observar los elementos antes referidos: al inicio del nodo, el nombre con el que se etiquetará la expresión 'LOCVLI' (Locución Verbal de Clase 1); luego, entre paréntesis, la variable V, con la que se detectará el verbo nuclear de la locución y, por último, en CI, las combinatorias de elementos que pueden componer la parte fija de la locución.

La gramática toma la información declarada en el diccionario electrónico, que contiene los lemas declarados de la siguiente manera:

agarrar, $\mathrm{V}+\mathrm{FLX}=\mathrm{AMAR}+\mathrm{Cl}=$ papa $+\mathrm{FXC}$

agachar, $\mathrm{V}+\mathrm{FLX}=\mathrm{AMAR}+\mathrm{DETCl}=\mathrm{el}+\mathrm{Cl}=\mathrm{moño}+\mathrm{FXC}$

echar, $\mathrm{V}+\mathrm{FLX}=\mathrm{AMAR}+\mathrm{CONTRC} 1=\mathrm{al}+\mathrm{Cl}=$ agua $+\mathrm{FXC}$

caer, $\mathrm{V}+\mathrm{FLX}=\mathrm{CAER}+\mathrm{PREPCl}=\mathrm{de}+\mathrm{Cl}$ =cajón+FXC

El lema se inicia con el verbo de la locución, seguido de su modelo de flexión (FLX=); posteriormente, se indican los elementos que constituyen la parte invariable. Todo esto debe llevar al final el comando $+\mathrm{FXC}$ para indicar que se trata de una expresión fija (frozen expression).

Con todo esto, es posible lograr una detección incluso en aquellos casos en los que la locución tenga los elementos separados, como en 'dejó Juan la mansa escoba':

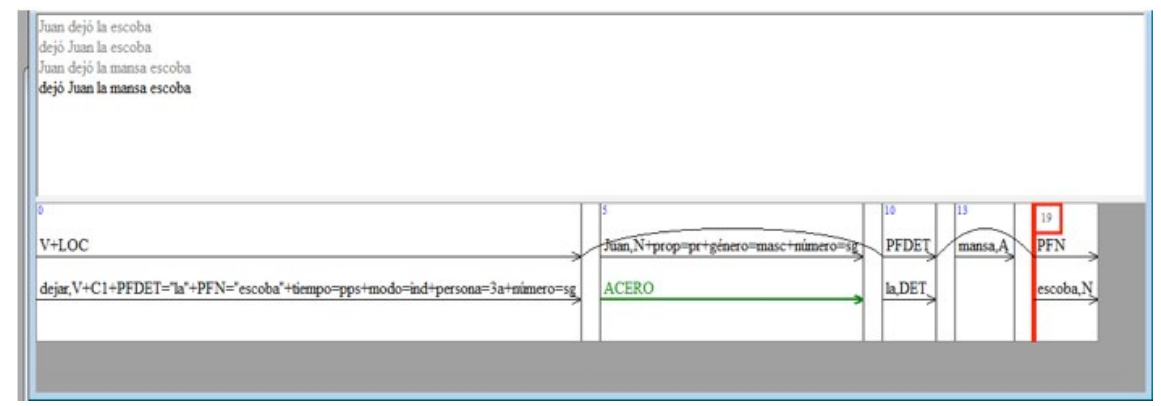

Figura 3. Ejemplo de detección de locución clase 1

La detección presenta "saltos" para unir los elementos de la LV que se encuentran separados. 
En relación con la clase 2, la gramática sintáctica tiene la siguiente estructura.

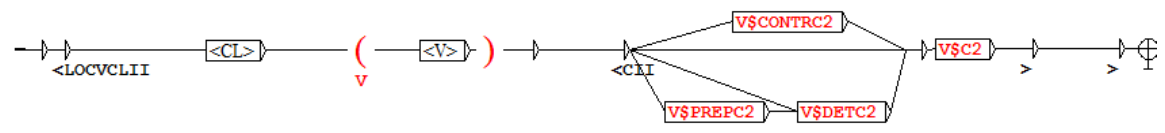

Figura 4. Gramática léxica para detectar locuciones clase 2

Como se puede apreciar, la única diferencia de esta gramática con la de clase 1 es la inclusión del pronombre clítico antepuesto a la unidad verbal. Esta información también debe declararse en las entradas del diccionario. De este modo, en el momento de realizar la detección, NooJ discrimina entre los elementos que poseen un CL, como en este caso, de los que no, como en el caso anterior.

$$
\begin{aligned}
& \text { abrir,V+FLX=PARTIR+CL+C2=cancha+FXC } \\
& \text { afirmar,V+FLX=AMAR+CL+DETC2=los+C2=pantalones+FXC } \\
& \text { agarrar,V+FLX=AMAR+CL+CONTRC } 2=\text { del }+\mathrm{C} 2=\text { moño+FXC } \\
& \text { caer, } \mathrm{V}+\mathrm{FLX}=\mathrm{CAER}+\mathrm{CL}+\mathrm{PREPC} 2=\mathrm{de}+\mathrm{C} 2=\text { maduro+FXC }
\end{aligned}
$$

Del mismo modo, en la figura 5 se puede evidenciar un ejemplo de detección. A diferencia del caso anterior, en esta oportunidad se han cargado los diccionarios de clase 1 y clase 2 para demostrar que la información declarada posibilita que NooJ discrimine adecuadamente entre un tipo de locución y el otro. 


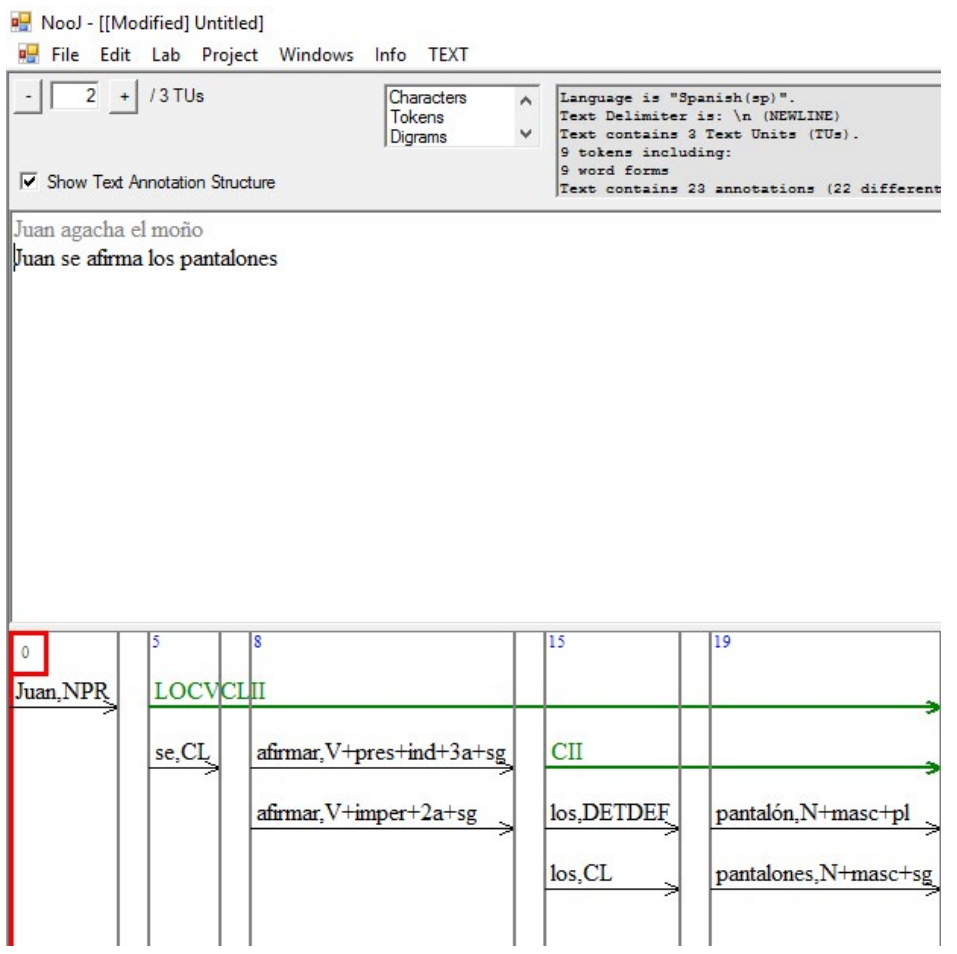

Figura 5. Ejemplo de detección de locución clase 2

Incluso si una locución de clase 1 admite un pronombre clítico antepuesto al verbo, lo que se asemejaría a la gramática de la clase 2 , NooJ realiza la detección de manera adecuada sin mezclar la información declarada en las gramáticas. Al igual que en el ejemplo anterior, en la figura 6 se muestra la detección realizada empleando los diccionarios con las respectivas gramáticas de las clases 1 y 2. 


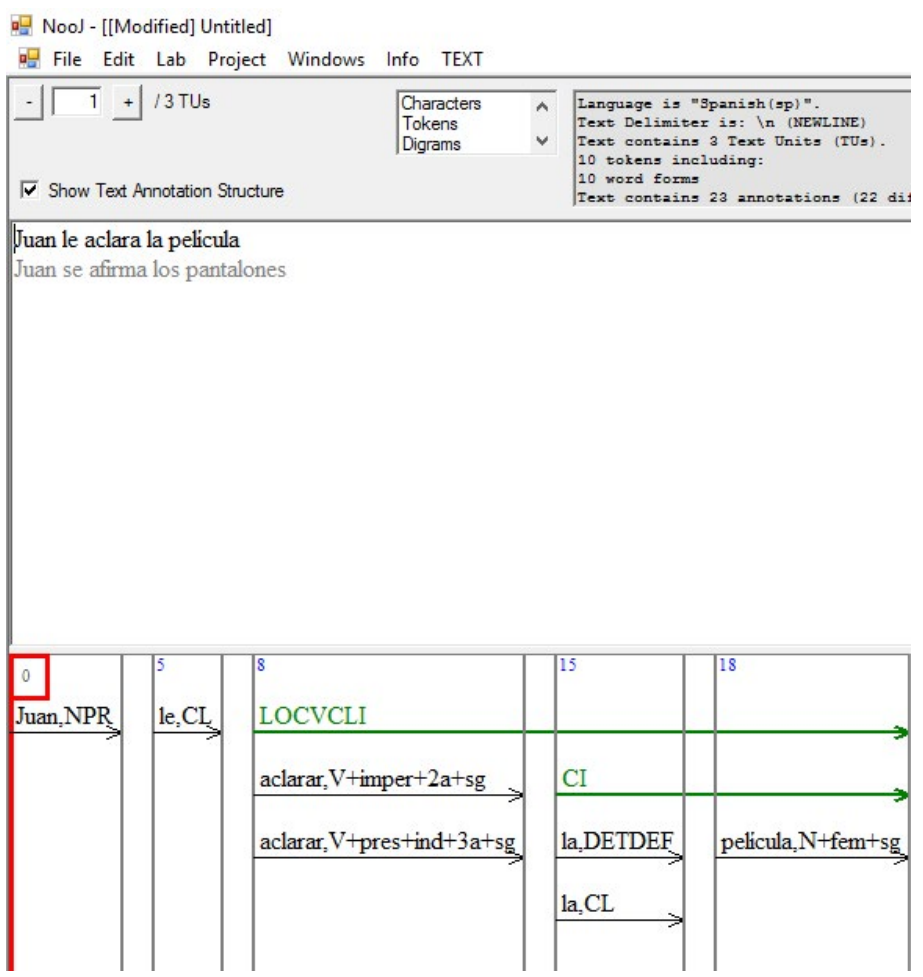

Figura 6. Ejemplo de detección locución Clase 1 que admite pronombre

Esta metodología fue probada en un corpus compuesto por textos extraídos de la prensa chilena. En la sección siguiente, se presentan los resultados obtenidos.

\section{Resultados obtenidos}

Para llevar a cabo la detección automática se cargaron en NooJ los diccionarios correspondientes a cada una de las clases de locuciones con sus respectivas gramáticas. En la figura 7 se presenta un fragmento del output realizado por software. 


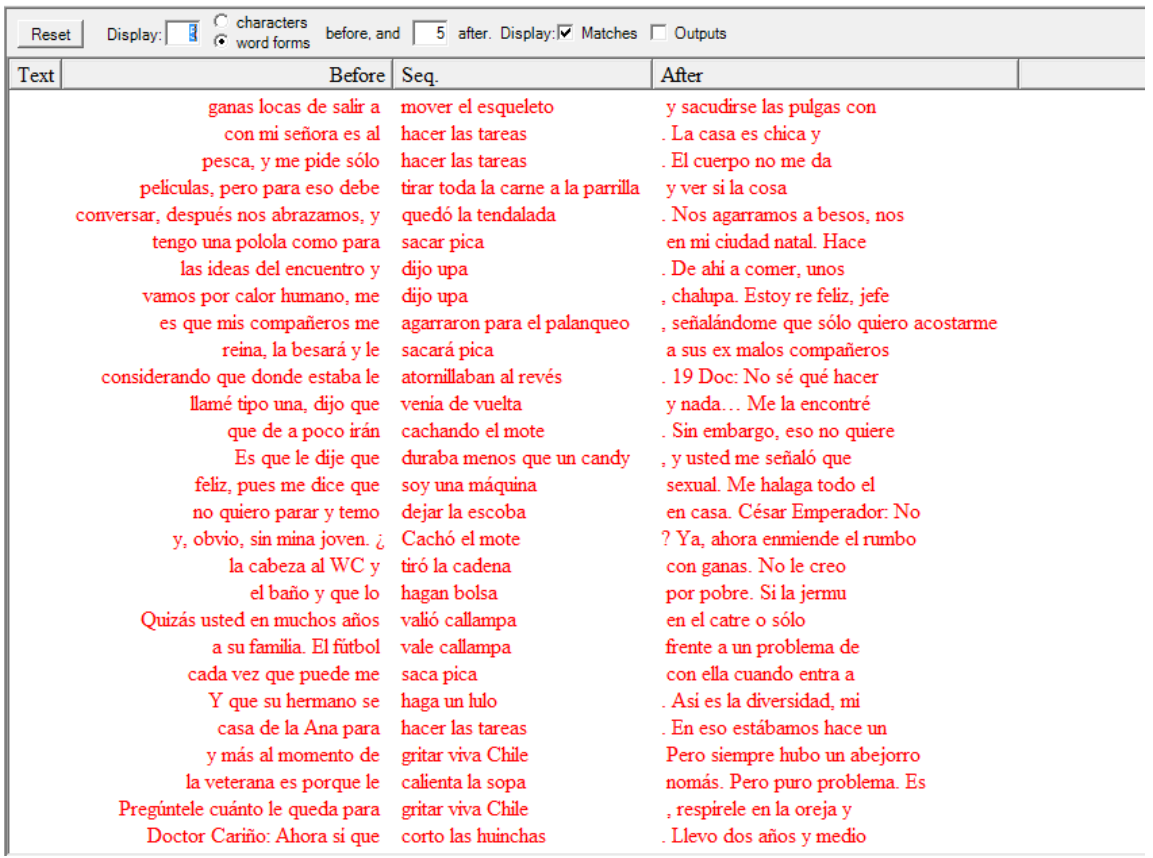

Figura 7. Ejemplo de detección de locuciones clase 1

El corpus empleado para poner a prueba la detección automática proviene del periódico nacional La Cuarta. De este medio se extrajeron textos de una de sus secciones denominada "Ventanita sentimental". En este espacio, los lectores envían cartas con diversas inquietudes. Se seleccionó este apartado debido a que esta sección se caracteriza por emplear un registro informal, y por ello se tuvo como supuesto que podrían ser textos ricos en modismos y locuciones verbales como las que se trabajan en esta investigación. En total, el corpus se compone de 33.094 palabras, lo cual equivale a más de cien cartas con sus respectivas respuestas.

Para la obtención de resultados se contabilizaron manualmente las LLVV incluidas en el corpus y se comparó con el output de NooJ. Con ello, se calcularon los porcentajes de precisión, cobertura y medida F. Vale aclarar que, en esta ocasión, no se tuvieron en cuenta aquellas locuciones del corpus que presentaban la estructura verboide más enclítica ('sabiéndoselas por libro'), 
quedando el análisis de estos para etapas futuras de trabajo. En la tabla 11 se listan los porcentajes logrados por el algoritmo.

\begin{tabular}{|l|c|}
\hline \multicolumn{1}{|c|}{ Resultados } & Resumen \\
\hline Locuciones por clase & 103 \\
\hline Detectó bien & 95 \\
\hline Detectó mal & 3 \\
\hline Detección total & 98 \\
\hline Cobertura & $92,23 \%$ \\
\hline Precisión & $96,94 \%$ \\
\hline Medida F & $94,53 \%$ \\
\hline
\end{tabular}

Tabla 11. Resultados detección por total

Puede observarse que los resultados obtenidos fueron positivos. Esto permite comprobar que la formalización realizada a partir del aparato metodológico que entrega la léxico-gramática fue adecuada. Sin embargo, debido a la limitada cantidad de clases que presentaba el corpus, no se pudieron validar los recursos construidos para las ausentes. Esto implica que la compilación de un nuevo corpus que contenga locuciones de todas las clases se constituye como un desafío, necesario para el estudio de este tipo de expresiones.

En relación con los resultados obtenidos por clase, se puede indicar que la mayor cantidad de locuciones se concentran en la clase 1 y 2 . Este resultado no llama lo atención considerando que más del 50\% de los lemas o entradas en el DUECh pertenecen a estas clases, por lo que se constituyen como locuciones diversas y a la vez comunes en el uso del español chileno. En cuanto a los porcentajes obtenidos, se puede observar que los resultados más descendidos se encuentran en la cobertura, lo que implica que para próximos trabajos se requerirá realizar gramáticas en las que se incluyan más propiedades de las locuciones. 


\begin{tabular}{|l|c|c|c|c|}
\hline \multicolumn{1}{|c|}{ Resultados } & Clase 1 & Clase 2 & Clase 4 & Clase 5 \\
\hline Locuciones por clase & 61 & 47 & 3 & 2 \\
\hline Detectó bien & 52 & 39 & 3 & 1 \\
\hline Detectó mal & 1 & 2 & 0 & 0 \\
\hline Detección total & 53 & 41 & 3 & 1 \\
\hline Cobertura & $85,25 \%$ & $82,98 \%$ & $100 \%$ & $50 \%$ \\
\hline Precisión & $98,11 \%$ & $95,12 \%$ & $100 \%$ & $100 \%$ \\
\hline Medida F & $91,23 \%$ & $88,64 \%$ & $100 \%$ & $66,67 \%$ \\
\hline
\end{tabular}

Tabla 12. Resultados detección por clase

Del mismo modo, se deberán revisar las cualidades declaradas en los diccionarios para detectar aquellas 9 locuciones que quedaron fuera en el caso de la clase 1, 8 en relación con la 2 y 1 en la clase 5 . No obstante, se puede señalar que los resultados son satisfactorios como primera aproximación al fenómeno de las LLVV en el español de Chile.

\section{Conclusiones}

Para concluir, cobra relevancia recordar los objetivos generales propuestos: (i) describir las propiedades morfosintácticas y la estructura argumental que proyectan las LLVV del español de Chile y (ii) detectar LLVV del español de Chile en textos de lenguaje natural. Estos dos grandes propósitos guiaron el quehacer investigativo y, a partir de los resultados evidenciados en el apartado anterior, se puede señalar que se consiguieron ambos. A este respecto, es importante indicar la efectividad del aparato metodológico que aporta la léxico-gramática en trabajos descriptivos como este, pues, por medio de las tablas, se pueden extraer las cualidades de las locuciones y, a su vez, se permite formalizar las estructuras para poder llevar a cabo la modelización computacional.

Así, por un lado, fue posible develar que, en la mayoría de los casos, las locuciones requieren de un argumento N0 +Humano. También se observó que, en relación con el uso de pronombres, un amplio porcentaje se emplea con pronombres dativos ('Juan le da bola a María'). En cuanto a los elementos "fijos", se pudo determinar que, en la mayoría de las clases, estas unidades 
pertenecen a sintagmas determinantes, nominales o preposicionales. Y, por último, se dio cuenta de la poca recurrencia al empleo del argumento N1, ya sea introducido o no por preposiciones.

Esta descripción posibilitó la formalización de estas unidades para realizar la posterior detección computacional. Al respecto, se obtuvieron resultados positivos en cuanto a la detección, pues todos los indicadores, ya sea, precisión, cobertura y medida F, estuvieron por encima del 80\%. Esto, gracias también a las propiedades que posee el software, ya que la elaboración del diccionario ofrece la opción de introducir un comando especial (+FXC) para el tipo de unidades que se desea detectar.

Por otro lado, se observó que, de las trece transformaciones contempladas en este estudio descriptivo, existen tres que son recurrentes de manera transversal a las clases. Asimismo, cobra relevancia indicar el alto promedio de transformaciones que admiten las locuciones de cada clase, pues eso da cuenta de que estas unidades son ricas en cuanto a propiedades sintáctico-semánticas y morfológicas y, por tanto, pese a que tradicionalmente fueron consideradas como invariables, se debe renovar esta visión.

Ahora bien, a pesar de que, en general, se puede señalar que se lograron los objetivos propuestos, algunos aspectos que quedaron fuera del alcance de esta investigación deberán ser abordados en un futuro. Al respecto, queda como proyecto la elaboración de tablas léxico-gramática para las locuciones pertenecientes al segundo grupo, esto es, las que en su entrada del diccionario poseen el adverbio de negación "no".

Asimismo, también se plantea para un trabajo posterior, la elaboración de reglas gramaticales que permitan la detección de locuciones que se emplean con uno o dos pronombres enclíticos en la unidad verbal ('hacérselo pebre'). Del mismo modo, cobra relevancia, como ya se mencionó anteriormente, tanto la inclusión de más transformaciones a la tabla, como la formalización de estas para enriquecer el procedimiento de detección. Para ello, se podría requerir de una mayor descripción de los elementos constitutivos de las locuciones, lo que se traduce en una descripción más detallada de las propiedades que estas unidades poseen.

En síntesis, el trabajo con vistas al futuro, en relación con el aspecto descriptivo, se organiza en torno a los siguientes ejes: (i) profundizar la descripción lingüística realizada en las tablas léxico-gramática para mejorar la 
detección automática; (ii) extender las posibilidades transformacionales que poseen las LLVV del español de Chile, y (iii) elaborar tablas para locuciones cuyo lema en el DUECh contenga el adverbio de negación "no". En cuanto al trabajo computacional, se pretende: (a) ampliar el diccionario electrónico de las LLVV; (b) enriquecer las gramáticas informáticas, y (c) elaborar gramáticas para la generación automática de oraciones que contengan una LV como predicado. Asimismo, también se incluye dentro de las tareas que se deberán realizar la compilación de un corpus mayor para poder probar la totalidad de los recursos elaborados.

\section{Referencias bibliográficas}

Academia Chilena de la Lengua. (2010) Diccionario de uso del español de Chile. Santiago: MN Editorial Ltda.

BONINO, Rodolfo. (2015) "Una propuesta para el tratamiento de los enclíticos en NooJ." Infosur Revista 7, pp. 31-40.

CHOMSKY, Noam. (1957) Syntactic structures. Berlín: Mouton \& Co.

CORPAS PASTOR, Gloria. (1996) Manual de fraseología española. Madrid: Gredos. Gross, Maurice. (1975) Méthodes en syntaxe. París: Hermann.

Gross, Maurice. (1984) "Lexicon-grammar and the syntactic analysis of French." En: Association for Computational Linguistics (1984). Proceedings of the 10th International Conference on Computational Linguistics and 22nd anual meeting on Association for Computational Linguistics. Association for Computational Linguistics, pp. 275-282.

HARRIS, Zelig. (1970) Papers in structural and transformational linguistics. Dordrecht: Springer.

MENA MARTínEZ, Florentina. (2003) "En torno al concepto de desautomatización fraseológica: aspectos básicos." Tonos Revista electrónica de estudios filológicos 5. Versión electrónica: <https://www.um.es/tonosdigital/znum5/estudios/H-Edesautomatizacion.htm>

ELIA, Annibale; Mario Monteleone \& Federica Marano. (2011) "From the concept of transformation in Harris and Chomsky to the Lexique-grammaire of Maurice Gross." En: Kasevich, Vadim; Yuri Kleiner \& Patrick Sériot (eds.) (2011). History of Linguistics. Amsterdam: John Benjamins, pp- 76-82.

PenAdÉs, Inmaculada. (2012) Gramática y semántica de las locuciones. Alcalá de Henares: Servicio de Publicaciones de la Universidad de Alcalá. 
PIVAUT, Laurent. (1989) Verbes supports et vocabulaire technique: sport, musique et activités intelectuells. Paris: Universidad de París. Tesis doctoral inédita.

SÁEz, Leopoldo. (2012) "El léxico del dialecto chileno: Diccionario de uso del español de Chile DUECh." Estudios filológicos 49, pp. 137-155.

Silberztein, Max. (2003) NooJ Manual. Versión electrónica: <http://www. NooJ4nlp.net/>

SilberzTEIn, Max. (2016) Formalizing Natural Languages: The NooJ Approach. Londres: Wiley Eds.

SILVAGNI, Federico. (2017) Entre estados y eventos. Un estudio del aspecto interno del español. Barcelona: Universidad Autónoma de Barcelona. Tesis doctoral inédita.

TOLONE, Elsa. (2012) "Conversión de las tablas del léxico-gramática del francés en el léxico LGLex". Ponencia presentada en el 2nd Argentinian Workshop on Natural Language Processing (WNLP'11). Córdoba: Universidad Nacional de Córdoba.

VIETRI, Simonetta. (2014) Idiomatic Constructions in Italian. A Lexicon-Grammar Approach. Amsterdam Netherlands: John Benjamins BV.

ZuluaGA, Alberto. (1975). "La fijación fraseológica." Thesaurus 30, pp. 235-288.

ZuluagA, Alberto. (2001) "Análisis y traducción de unidades fraseológicas desautomatizadas." PhinN 16, pp. 67-83.

\section{NOTA BIOGRÁFICA / BIONOTE}

WALTER KOZA es Doctor en Humanidades y Artes con Mención en Lingüística, egresado de la Universidad Nacional de Rosario (Argentina). Actualmente, es Profesor Auxiliar de la Pontificia Universidad Católica de Valparaíso (Chile). Sus áreas de investigación son la lingüística computacional, los estudios del léxico y la gramática del español.

WALTER KOZA is PhD in Humanities and Arts with mention in Linguistics graduated of Universidad Nacional de Rosario (Argentina). Nowaday is Assistan Professor at Pontificia Universidad Católica de Valparaíso (Chile). His research areas are computational linguistic, the lexicology and Spanish grammar. 
NATAlia Rivas es Profesora de Castellano y comunicación y Licenciada en Lengua y Literatura Hispánica, egresada de la Pontificia Universidad Católica de Valparaíso (Chile).

NATALIA RIVAS is Teacher of Spanish and Comunication and Bachelor in Language and Hispanic Literature, graduated at Pontificia Universidad Católica de Valparaíso (Chile). 